\title{
MARK III VLBI OBSERVATION OF PULSARS ${ }^{+}$
}

N. Bartel ${ }^{1}$, R.J. Cappallo ${ }^{2}$, M.I. Ratner ${ }^{1}$, A.E.E. Rogers ${ }^{2}$, I.I. Shapiro ${ }^{1}, A . R$. Whitney

$1_{\text {Harvard-Smithsonian Center for Astrophysics }}$ 2

NEROC Haystock Observatory

\section{INTRODUCTION}

Mark III VLBI observations of the pulsars PSR $0329+54$ and PSR $1133+16$ were made at $2.3 \mathrm{GHz}$ using antennas with diameters and locations as follows: 100m, Effelsberg, West Germany (but only for SPR 0329+54); 43m Green Bank, WV, USA; and 40m, Big Pine, CA, USA. The Mark III processor at the Haystack Observatory was "gated" to compute visibility amplitudes and phases as a function of pulsar longitude. This method allowed a) an improvement of the signal to noise ration, by as much as a factor of ten in the case of PSR 1133+16, and b) an interferometric investigation of the pulse structure.

\section{PULSAR ASTROMETRY}

Observations of the pulsars and of compact extragalactic sources $0355+508$ (NRAO 150) and 1119+183, each nearby in the sky to one of the pulsars, yielded the following positions (epoch 1981.21) and uncertainties given as the sum of our estimates of sistematic errors:

PSR 0329+54: $\Delta \alpha($ VLBI-PTA $)=00^{5} 3 \pm$

PSR 1133+16: $\alpha(1950)=11 \mathrm{~h} 33^{\mathrm{m}} 27.5409 \pm_{0} \mathrm{~s}_{03}, \delta(1950)=16^{\circ} 07^{\prime} 38^{\prime \prime} .9 \pm_{0}{ }^{\prime \prime} .3$. These positions differ from those determined from pulse-time-of arrival measurements (PTA) by Manchester and Taylor (A.J.86, 1953, 1981) as follows:

PSR 0329+54: $\alpha(1950)=03^{\mathrm{h}} 29^{\mathrm{m}} 11$ s.0133 $\pm_{0} \mathrm{~s} 0005, \delta(1950)=54^{\circ} 27^{\prime} 37^{\prime \prime} .300 \pm 0 " .005$ PSR 1133+16: $\Delta \alpha($ VLBI-PTA $)=0$ s $056 \pm 0$ s $008, \Delta \delta($ VLBI-PTA $)=-0 " .9 \pm 0 " .3$.

The errors shown are the root-sum-squares of the errors given above and the statistical standard errors given by Manchester and Taylor. The differences between the VLBI and PTA values, if significant, may be due most1y to the difference in orientation of the VLBI reference frame from that in which the timing positions are expressed.

3. UPPER LIMIT OF APPARENT PULSAR SIZE

In order to account for the total flux density variations of PSR +54 , the correlated flux densities of all three interferometers were divided by the corresponding correlated flux densities for the interferometer with the shortest baseline. The width of a circular Gaussian determined by a least-squares fit to these normalized amplitudes is $0.23 \pm 8: 13$ mas, where + Discussion on page 455 
the error was estimated by varying the gain of each station independently by $15 \%$.

\section{UPPER LIMIT OF OFF-PULSE CONTINUUM EMISSION}

The data for PSR $0329+54$ were recorrelated using a gating window corresponding to 0.8 of the pulsar period, centered between the pulses. All of these "off-pulse" data were coherently integrated ( $\sim 2.9$ hours total) by using the technique of phase referencing. No continuum emission was detected at the position of the pulsar above a correlated flux density level of $2.5 \mathrm{mJy}(3 \sigma)$. A separate 1 imit was determined for the approximately two minute period during which the received pulses were strongest. This limit is less than 0.1 percent $(4 \sigma)$ of the flux density of the peak of the average pulse during that period.

5. INTERFEROMETER PHASE WITHIN THE PULSE STRUCTURE

In order to search for position offsets between the emission regions of the three components in the average pulse profile of PSR 0329+54, the Green Bank-Effelsberg interferometer phase-delays for these components

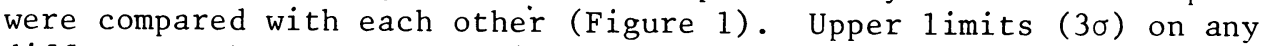
differences between the positions of the three components are:

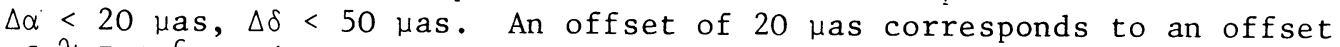
of $\approx 7 \times 10^{6} \mathrm{~km}$ (less than five solar diameters)at an assumed distance of $2.3 \mathrm{kpc}$ for PSR 0329+54. Also no significant phase differences were detected within individual pulses, which were partitioned into 75 bins each of $715 \mu \mathrm{s}$ extent.
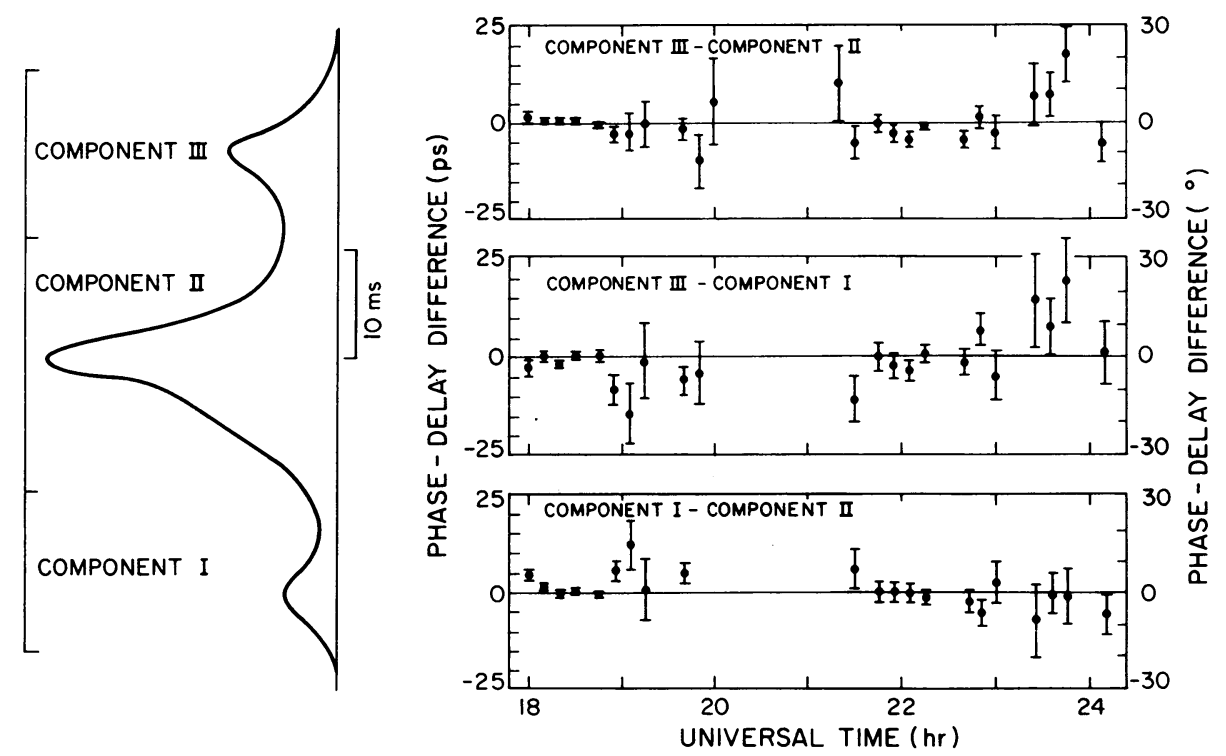

Figure 1: Differences of interferometer phases for each pair of components in the average pulse profile of PSR 0329+54 from observations with the Green Bank and Effelsberg antennas. Error bars shown signify standard deviations (statistical). 